\title{
Role of Sport Public Relations for the COVID-19 Outbreak: Interview With Kwon Heo, Public Relations Manager at Kia Tigers
}

\author{
Sungwook Son, Antonio S. Williams, and Yoon Heo \\ Indiana University Bloomington
}

Following a successful flattening of the curve in terms of the spread of COVID-19, the Korean Baseball Organization (KBO) became one of the first professional-level sport leagues to start its season in early. With limited programming available due to various sports cancellations and postponements, ESPN made the decision to broadcast the Korean baseball games. With this in mind, the perspective of a practitioner in Korean baseball could be valuable for numerous sport experts in other countries. Hence, an interview with Kwon Heo, a public relations (PR) manager with the Kia Tigers, was conducted. Heo entered the sport industry as a reporter for Gwangju daily newspapers from 1995 to 2001. Since 2002, he has been working as the head of PR for the Tigers. The Kia Tigers are the most successful professional baseball team in Korea, with 11 Korean series titles, and Kwon is responsible for the overall planning and promotion of the franchise. He also earned a doctorate in sports marketing from Mokpo National University in 2013, and since 2009 he has been teaching sports promotion, sports media, and sports marketing to students as a part-time lecturer at Mokpo National University in order to deliver his field experiences to students. He has also been in charge of conducting lectures regarding the training of sports industry personnel at universities near the region (e.g., Chosun University and Sunchon University).

Son, Williams, and Heo: What kind of work have you been doing for Kia Tigers? Could you describe the overall work of the Tigers PR team and your daily responsibilities as a team leader?

Heo: Since 2002, I have been in charge of PR, particularly media promotion. I am responsible for the team's mid- to long-term vision development. My daily tasks also include active promotion of the team and players. The main duties of the PR team this year include press publicity, managing the franchise's social media channels, and production of promotional materials. As the team leader, I also serve as the spokesperson for the team. 


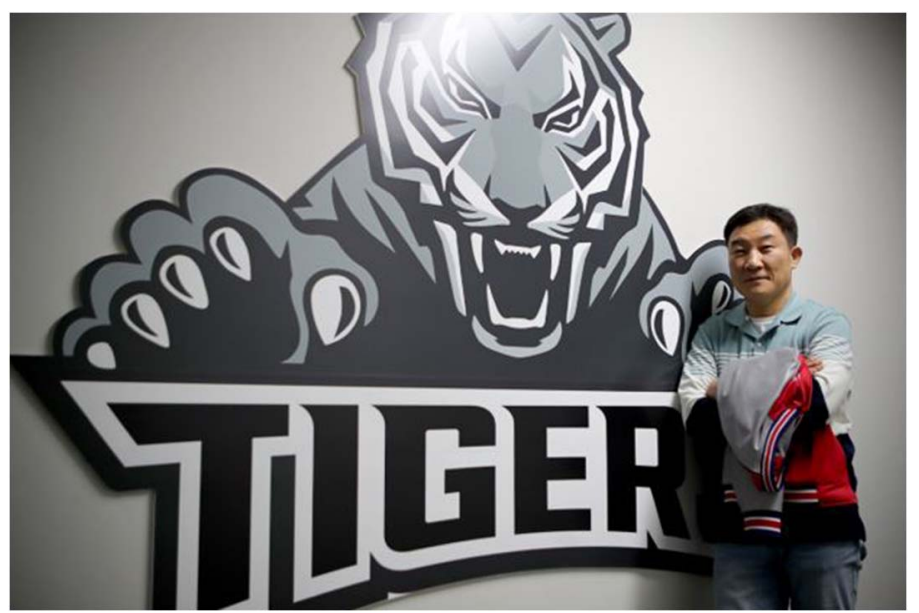

Kwon Heo-Public relations manager, Kia Tigers.

Son, Williams, and Heo: You have mentioned that your duties include promotion of both the team and players. Are you also in charge of promoting players?

Heo: Player-related PR includes advertising our players. It covers various promotional activities, from developing a new star player to promoting individual players by arranging multiple media exposure. Hence, I work closely with the Tigers players as a PR agent. Regarding COVID-19, I was touched by how the players decided to wait for training at their residences in Gwangju, where the home stadium is located, rather than to return home to take a few days off. At that time, there was a rapid increase in the number of confirmed cases across the country and they tried their best to prevent any disease transmission to their family members. Also, the foreign players' reaction to the COVID-19 incident was unforgettable. Many Korean professional baseball teams sent foreign players back to their home countries after overseas spring camps. That was because Korea was experiencing a surge in the number of confirmed COVID-19 cases during that time. Of course, after some time, foreign players who thought Korea was safer, returned to Korea in a hurry. However, our team's foreign players, manager Matt Williams, and coach Mark Weidemaier came back to Korea with the team right after the Florida spring camp, which gave great trust to the front office officials and our fans.

Son, Williams, and Heo: You have diverse work experience. How did you get into the field of sport PR?

Heo: I started my professional career as a newspaper reporter. During my career, I became a sportswriter reporting on professional baseball. When the team was rebranded from the Haitai Tigers to the Kia Tigers, I got scouted by the franchise as a sport PR specialist. That was in 2002, and I have been working in the field of sport PR since then.

Son, Williams, and Heo: As an industry expert, can you explain the impact of COVID-19 on Korean baseball and the Tigers? What has changed? What difficulties is the industry facing at this moment? 
Heo: COVID-19 has influenced many aspects of our lives, especially the world of sports, which typically involves large crowds including fans and players. Notably, the situation has had an immeasurable impact not only on Korean professional sports but also on amateur sports, leading to significant economic losses and poor performance due to a lack of training.For KBO teams, there are many things that have temporarily changed. First of all, the league's altered schedule is the biggest change. The KBO has canceled the spring exhibition matches for the first time since the launch of the league. Fortunately, our season will start soon, although the games will be played without any audience. The league, of course, will keep following a strict social distancing campaign throughout its 144 regular-season games. I believe this has become possible due to the social atmosphere and the national policies of Korea, gradually easing lockdown restrictions and allowing us to return to our daily lives.

Another thing that changed due to COVID-19 is fan service. As a precaution, we are planning to conduct games without spectators. Also, any promotional event in which contact between players and fans would occur at close range, such as a meet and greet, has been banned. Importantly, there have also been various changes in the lives of our players. When entering public spaces such as the stadium, locker room, or team transportation, players must have their temperature checked and must sanitize their hands. Hygiene management, which is a new task for us, has also been strengthened, making players and their families refrain from going out. It is extremely hard for our staff and players to be restrained from social activities for months.

We also changed our seating arrangements in a zigzag way in the team cafeteria and meeting room. After-game dinners have also been banned to comply with national social distancing policies. The work environment for the team staff has been changed as well. The employees have been distributed into three different offices to prepare for any emergency. Particularly for the players, some actions on the field, such as high-fives with bare hands, spitting, and chewing tobacco and sunflower seeds, have been banned. The whole typical baseball scene has been changed due to the current situation with COVID-19.

Again, one of the hardest parts for us is the changed schedule. The players are exhausted from several months of training, and the staff is also tired from spending all of their days preparing for the upcoming season. Even if the season starts, it would be really hard to play ball games without hearing the cheering of the crowd.

In terms of team management, there is no revenue from multiple sources, such as ticket sales. Operating costs, however, have increased due to preventive measures against COVID-19. What is more concerning is that bigger problems may arise when the no-audience restriction is lifted. Although we are thoroughly managing the prevention of epidemics and players are going through COVID-19 prevention education, we are still worried about the possibility of infections at the stadium. The league may face an indefinite shutdown if even one confirmed COVID-19 case occurs at any baseball park.

Son, Williams, and Heo: What do you think the core role of sport PR is, particularly when direct communication with fans is difficult, as during these days?

Heo: The franchise is currently contemplating on how to earn fans' understanding regarding the situation and resolve their thirst for baseball by looking into their 
wants and needs. Because fans cannot watch baseball at the stadium, we are trying to create new team-related content to entertain fans at home. Above all, because coverage activity is limited, various news items about the team are not getting delivered to fans. The PR team is now focusing on finding new coverage items and spreading them through both traditional media and social media channels. Social media is especially utilized to communicate with fans and this has helped fans to better understand the current situation and support the team's efforts against COVID-19.

We now actively produce and distribute promotional digital content, such as video clips, so that fans can access to them in their homes. Every press release, official statement, or promotional information is distributed via digital media platforms. In addition, through numerous online media channels owned by the franchise, we aim to create various types of novel content, such as card news or infographics. Sport PR channels have recently been changed from traditional media to digital media owned by the team, such as the official Tigers YouTube channel or social media accounts, and COVID-19 has actually accelerated this change.

I believe that these channels allow us to get closer to our fans. Feedback comes right away and direct communication with fans also has become possible. It is very effective in terms of the promotion and marketing of the team. By sharing diverse team-related information, we can increase fan loyalty and maintain a lasting consumer-brand relationship. Our team currently operates diverse media platforms, including the team's website, blogs, chatting apps, social media platforms, and video channels. Since the COVID-19 outbreak, the amount of content that the team uploads, as well as the number of views, has drastically increased.

Son, Williams, and Heo: You have mentioned that fan feedback comes right away via digital channels. We are curious as to whether you refer to the comments and opinions of the fans when establishing promotions strategies.

Heo: We refer to various fan postings on the Tigers' website, social media channels, and even online baseball fan communities when developing our PR strategy. For example, when fans send us feedback on the content they want, we try our best to produce the exact content in various formats. Broadcasting intra-squad games via YouTube is a perfect example of content devised from fans' active requests.

Son, Williams, and Heo: Regarding the broadcast of intra-squad games via YouTube, this has become a huge issue among the U.S. baseball fans. Could you explain this is detail?

Heo: The broadcasting of intra-squad games on YouTube has been going on for years. Beginning with fans' continuous requests, the Kia Tigers have been broadcasting camp practice games and intra-squad games on our official YouTube channel. I think the reason why it has become a hot topic in the United States this year is because Korea has responded well to the pandemic and we were able to conduct team training sessions and practice games at a time when all professional events around the world were being suspended. 
For this year, all the KBO franchises agreed to broadcast exhibition games on YouTube for fans who were thirsty for baseball. It was also planned in order to satisfy fans who were not able to come to stadiums due to required social distancing. In order to strengthen the fan experience with the YouTube broadcast, our manager Matt Williams, pitching coach Jae-Weong Seo, hitting coach HeeSeop Choi, and defensive coach Min-Ho Kim appeared as daily commentators. Moreover, our star players, team scouts, and staff also participated, sitting at the broadcasting booth to directly answer questions from fans and communicate with them in real time. This has drawn extremely positive responses from our fans.

Son, Williams, and Heo: What did you feel about the PR initiatives during this COVID-19 incident? Could you tell me what you learned from the current situation?

Heo: The biggest thing, of course, is the importance of fans. It is extremely regrettable that COVID-19 has banned all contact with our fans, but it was an inevitable choice in the current situation, and I was grateful that fans kindly understood and showed their endless support. Also, it is very sad that we have to play games without fans and spectators because they are an important part of baseball games. Furthermore, I realized the need of sport PR once again. We need to work hard to quench fans' thirst for baseball through various content. I also noticed the importance of the role of the government and the social system in the society. Thanks to the government's effective quarantine system, we were able to have team training sessions and play baseball games safely in this uncertain time.

Son, Williams, and Heo: Interestingly, you mentioned the role of the government. The Korean government's effective response to COVID-19 is receiving a lot of attention from other nations. Has the government's policy had any impact on the promotion of the Tigers? What is the overall KBO response to these policies?

Heo: The government's new COVID-19 policies are affecting the KBO a lot. Currently, the government has developed a social distancing campaign and other COVID-19 policies, and these have become the base of every decision made by the $\mathrm{KBO}$ board of directors and the general managers. Because professional baseball is an event in which a large number of people gather and there are hundreds of related personnel involved, the government's policy is an important criterion in every decision-making process of the KBO.

The KBO has developed an integrated COVID-19 response manual following the government-issued policies. By using this manual, the PR team of each franchise develops its own PR plans. For instance, any reporter who enters the stadium will go through a temperature check and must wear a face mask. They also have to set a safety distance of at least $2 \mathrm{~m}$ from the interviewer during interviews. These policies are all made based on the government-provided manual for COVID19. All KBO teams have agreed to these guidelines and have actively responded in order to prevent any infection in the Korean baseball community.

Son, Williams, and Heo: Do you communicate with other KBO teams regarding COVID-19 and the safety of the players and fans? If so, what kind of information or opinion do you share? 
Heo: All KBO franchises are cooperating and jointly responding to the current situation with the KBO executives. As I mentioned, every team is using the same integrated response manual developed by the $\mathrm{KBO}$, and each team is preparing for the upcoming season according to these guidelines. Whenever it is necessary, we share any valuable opinion and information through PR team leader meetings by holding video conferences.

Son, Williams, and Heo: It seems like many things have changed due to COVID19. How do you think the COVID-19 incident will affect the future of Korean baseball?

Heo: I think there will be a lot of impact. Considering the team's mid- to long-term development, I think it is time to prepare for the post-corona era. I believe the biggest change we will face is a change in lifestyle and spectator behavior. The culture of overtime work and company dinners, which has been very common in Korea, will disappear. The unique Korean baseball spectating culture, such as baseball field dinners, that has traditionally been the pleasure of office workers will eventually disappear. Watching baseball while having chimaek (fried chicken and beer) or singing player chants has been one of the best ways to relieve stress for many Korean baseball fans, but this will also go away. These changes in baseball consumption patterns will have a huge impact on the KBO league and franchises in general. One of the biggest revenue sources for Korean professional baseball teams, ticket sales, will decrease with the decline in spectators. Additionally, the instadium food and beverage business will also see a significant drop in sales. Because KBO teams still largely rely on these revenue sources, these revenue decreases may endanger the franchises. Thus, we are currently trying to solve this problem by developing additional revenue channels.

Son, Williams, and Heo: Korea's response to COVID-19 and the KBO's quick opening are gaining much attention worldwide. What kind of advice would you like to give to people in sports leagues and teams in other countries, or even to those in the field of sport PR?

Heo: I think the KBO's early season opening is one of the results derived from the Korean government's effective COVID-19 response system. I believe that the role of government is extremely significant for the sport industry. I also think that the voluntary and responsible behavior of players, fans, and staff are the most important factor. We all have been following the government-led policies and suggestions and everyone recognized the severity of the current situation and worked hard for a quick season opening. Thanks to that, we had good results. Furthermore, what I felt as a publicist throughout this situation is the importance of information transparency. By making all information transparent to the public, we can gain cooperation from the media and ask for fans' understanding. Because the COVID-19 situation is not over yet, I would appreciate it if the players, the front office, and the fans all behave responsibly to keep each other safe from the virus by following the social distancing campaign. 